\title{
Avaliação das ações do programa Hiperdia para pacientes hipertensos
}

Evaluation of the actions of the Hiperdia program for hypertensive patients

Evaluación de las acciones del programa hiperdia para pacientes hipertensos

Recebido: 02/01/2018

Aprovado: 12/06/2018

Publicado: 01/08/2018

\section{Anderson de Oliveira Vieira ${ }^{1}$ Fabiana Bernadelli de Andrade ${ }^{2}$ Gabriel Antônio Nogueira Nascentes ${ }^{3}$ \\ Dalmo Correia ${ }^{4}$ Marlene Cabrine dos Santos ${ }^{5}$}

Este é um estudo quantitativo, descritivo e exploratório com o objetivo de avaliar a influência das ações do HIPERDIA na qualidade de vida dos pacientes hipertensos de uma cidade do Triângulo Mineiro, realizado entre 2013 e 2016. Utilizou-se questionário sociodemográfico e de saúde, coletas de sangue, bem como intervenção educativa. Quatrocentos e cinco hipertensos $(18,4 \%$ do total dos cadastrados na cidade) participaram do estudo. Diabetes foi significativamente maior nos pacientes hipertensos $(41,98 \%)$ do que nos normotensos, assim como problemas visuais $(58,77 \%)$ e cardíacos $(26,17 \%)$. Em $61,14 \%$ dos casos, apesar da medicação, a pressão arterial não estava controlada. Outros fatores de risco relevantes foram: sedentarismo (63,46\%), sobrepeso e obesidade $(80,72 \%)$, e medida da circunferência abdominal $(88,3 \%)$. Conclui-se que os usuários do HIPERDIA apresentam fatores de risco para complicações isquêmicas que poderiam ser reduzidos com melhor adesão às práticas incentivadas pelo Programa.

Descritores: Sistema Único de Saúde; Centros de saúde; Fatores de risco; Hipertensão.

This is a quantitative, descriptive and exploratory study, aimed at evaluating the influence of the actions of the program HIPERDIA in the quality of life of hypertensive patients in a city in the Triângulo Mineiro, from 2013 to 2016. It used sociodemographic and health questionnaires, blood collection, as well as educational interventions. Four hundred and five hypertensive patients $(18.4 \%$ of those registered in the city) participated in the study. Diabetes was significantly higher among hypertensive patients $(41.98 \%)$ than in normotensive ones. The same was true for eyesight $(58.77 \%)$ and cardiac $(26.17 \%)$ problems. In $61.14 \%$ of cases, despite the medication, blood pressure was not under control. Other relevant risk factors were: sedentarism (63.46\%), obese and overweight patients (80.72\%), and abdominal circumference measurements (88.3\%). Users of the HIPERDIA have risk factors for ischemic complications that could be cut down if they adhered better to the practices encouraged by the Program. Descriptors: Unified Health System; Health centers; Risk factors; Hypertension.

Este es un estudio cuantitativo, descriptivo y exploratorio con el objetivo de evaluar la influencia de las acciones del HIPERDIA en la calidad de vida de los pacientes hipertensos de una ciudad del Triângulo Mineiro, realizado entre 2013 y 2016. Se utilizó un cuestionario sociodemográfico y de salud, colectas de sangre, así como intervención educativa. Cuatrocientos cinco hipertensos $(18,4 \%$ del total de los registrados en la ciudad) participaron en el estudio. La diabetes fue significativamente mayor en los pacientes hipertensos (41,98\%) que los normotensos, así como problemas visuales $(58,77 \%)$ y cardíacos (26,17\%). En el 61,14\% de los casos, a pesar de la mediación, la presión arterial no estaba controlada. Otros factores de riesgo relevantes fueron: sedentarismo $(63,46 \%)$, sobrepeso y obesidad $(80,72 \%)$ y medida de la circunferencia abdominal $(88,3 \%)$. Se concluye que los usuarios del HIPERDIA presentan factores de riesgo para complicaciones isquémicas que podrían ser reducidos con mejor adhesión a las prácticas incentivadas por el Programa.

Descriptores: Sistema Único de Salud; Centros de salud; Factores de riesgo; Hipertensión.

1. Biomédico. Especialista em Docência na Educação Superior. Mestre em Medicina Tropical e Infectologia. Uberaba, MG, Brasil. ORCID: 0000-0002-5760-5620 E-mail: anderson.ovieira@uol.com.br

2. Biomédica. Especialista em Saúde do Adulto pela UFTM. Uberaba, MG, Brasil. ORCID: 0000-0003-4847-3635 E-mail: bia_delli@hotmail.com

3. Biomédico. Mestre e Doutor em Medicina Tropical e Infectologia. Uberaba, MG, Brasil. ORCID: 0000-0002-8934-6619 E-mail: gabrielnog@yahoo.com.br

4. Médico. Especialista em Infectologia. Mestre e Doutor em Medicina Tropical. Professor Associado da UFTM. Uberaba, MG, Brasil. ORCID: 0000-0002-0209-7016 E-mail: dalmo@pesqpg.uftm.edu.br

5. Farmacêutica. Mestre em Ciências. Doutora em Medicina Tropical e Infectologia. Professora Adjunta da Universidade Federal do Triângulo Mineiro (UFTM). ORCID: 0000-0002-3288-3974 E-mail: marlenecabrine@yahoo.com.br 


\section{INTRODUÇÃO}

A hipertensão arterial sistêmica (HAS) é uma condição clínica multifatorial caracterizada por níveis elevados de pressão arterial (PA), sendo o diagnóstico estabelecido a partir da aferição da PA sistólica maior ou igual a $140 \mathrm{mmHg}$ e PA diastólica maior ou igual a $90 \mathrm{mmHg}$ sem uso de medicação anti-hipertensiva.

Tal doença é um grave problema de saúde pública e uma das doenças crônicas responsáveis por expressivas taxas de internação, custos elevados com a morbimortalidade associada à doença $\mathrm{e}$ comprometimento da qualidade de vida para os portadores, uma vez que sua progressão é lenta e contínua, geralmente fazendo com que os portadores somente apresentem sintomas quando problemas cardiovasculares já estão instalados ${ }^{1-4}$.

Sabe-se que é um dos fatores de risco mais prevalentes no desenvolvimento de doença arterial coronariana, acidente vascular cerebral, doença vascular periférica, insuficiência renal e insuficiência cardíaca congestiva.

Estima-se uma prevalência mundial de $26,4 \%$ de pacientes hipertensos. No Brasil a prevalência varia de 22,3 a 43,9\%, sendo que cerca de $30 \%$ dos óbitos por causas conhecidas são devido às doenças cardiovasculares ${ }^{1,4,5}$.

Em função da gravidade da HAS, o governo federal criou o programa HIPERDIA (Programa Nacional de Atenção à Hipertensão e Diabetes mellitus), um programa nacional de assistência aos hipertensos e diabéticos, que consiste em um sistema de cadastramento e acompanhamento contínuo desses indivíduos, através das Unidades Básicas de Saúde (UBS) de cada município, disponibilizando os medicamentos e equipes multiprofissionais para a realização de encontros periódicos sobre os cuidados referentes a alimentação, prática de atividade física e hábitos de vida saudáveis ${ }^{6,7}$.

A proposta do programa é reduzir o número de internações, a procura por prontoatendimentos, os gastos com tratamentos e complicações, aposentadorias precoces e mortalidade cardiovascular, com consequente melhoria da qualidade de vida dos portadores ${ }^{1}$.

Desde a implantação do programa em 2002, houve uma redução acentuada na incidência de hospitalização por acidente vascular encefálico isquêmico (AVEI) no Brasil ${ }^{6}$. Considerando que o programa HIPERDIA fornece informações/rotinas de hábitos de vida saudáveis para os seus usuários (hipertensos e/ou diabéticos) e que podem auxiliar na redução dos fatores de risco para os AVEI, este estudo tem como objetivo avaliar a influência das ações do HIPERDIA na qualidade de vida dos pacientes hipertensos de uma cidade do Triângulo Mineiro.

\section{MÉTODO}

Este é um estudo quantitativo, descritivo e exploratório, realizado nas Unidades Básicas de Saúde (UBS) da cidade de Uberaba, no período de agosto de 2013 a abril de 2016, com indivíduos hipertensos que fazem acompanhamento no programa HIPERDIA. Participaram também do estudo, voluntários, funcionários do Serviço de Patologia Clínica, HC-UFTM/ Filial EBSERH, classificados como normotensos.

Com vistas ao esclarecimento dos pacientes atendidos no HIPERDIA, nas diferentes UBS sobre saúde e HAS, palestras curtas e em linguagem fácil foram realizadas com os participantes. Essa atividade foi acompanhada por toda a equipe de saúde das UBS.

Todos os indivíduos do estudo concordaram e assinaram o termo de consentimento livre e esclarecido após informação dos objetivos da pesquisa e orientação em linguagem acessível.

Todos os pacientes/voluntários preencheram um questionário contendo dados sobre atividade física, fumo, etilismo, diabetes, problemas renais, dislipidemias, medicações em uso, obesidade, habitação em zona rural em algum momento, hábitos alimentares, além de dados pessoais como nome, RG, data de nascimento, gênero, endereço e telefone para contato. Foram obtidos também dados anteriores de ECG e 
Raios-X dos pacientes que já tinham realizado esse exame e que apresentaram o laudo.

A pressão arterial foi aferida de todos os pacientes, nas posições sentada e deitada, em ambos os braços, indicando parâmetros de bom controle se $\leq 140 \times 90 \mathrm{mmHg}$, seguindo as recomendações das 7a Diretriz Brasileira de Hipertensão Arterial (2016) ${ }^{4}$, com aparelho de pressão digital de pulso, marca G-Tech, modelo BP3AF1-3, lote 1710, certificado pelo Inmetro. 0 peso e a altura (para cálculo do IMC) e a medida da circunferência abdominal foram obtidos pelo cartão do usuário.

Os dados numéricos foram analisados por meio dos testes t ou Mann-Whitney, na comparação entre os grupos normotenso e hipertenso. Quanto aos dados categóricos, a comparação entre os grupos foi realizada por meio do teste do qui-quadrado. A análise estatística foi realizada com o auxílio do software Statistica 10.0 (Statsoft, Tulsa, OK, 2011) e foram considerados significativos os resultados que apresentaram $p$-value $<0,05$.

\section{RESULTADOS}

No período do estudo foram visitadas 24 das 26 unidades de atendimento ao público (92,3\%), incluindo unidades urbanas e rurais, divididas em UBS, Unidades Matriciais de Saúde (UMS) e Unidade de Saúde da Família (USF), distribuídas em três distritos da cidade.

Nas 110 visitas realizadas, a média de atendimentos foi de 20 pacientes participantes do HIPERDIA por dia. Nesse período foram abordados cerca de 2.200 hipertensos $(\mathrm{H})$, sendo que somente $18,4 \%$ $(n=405)$ aderiram ao projeto de pesquisa. Além desses, concordaram em participar do estudo 17 pessoas normotensas, que foram o nosso grupo controle para algumas comparações. Além dos pacientes, também participaram das ações de educação em saúde, os profissionais das equipes do HIPERDIA, cerca de 24 enfermeiros (as), 40 técnicos (as) de enfermagem e 120 agentes de saúde.

A avaliação dos dados dos questionários respondidos pelos pacientes do estudo mostrou que $61,7 \%(\mathrm{n}=250)$ dos hipertensos e 70,6\% (n=12) dos normotensos tinham menos de 60 anos de idade, com média de 56,93 anos para o grupo hipertenso e 52,47 anos para o grupo normotenso ( $p$ value $=0,118)$. Chamou a atenção o fato de $19,51 \%(n=79)$ dos pacientes hipertensos se encontrarem na faixa etária entre 36-50 anos e $3,95 \%(n=16)$ na faixa entre $26-35$ anos. Em relação ao gênero, $73,7 \%$ e $58,8 \%$ dos pacientes hipertensos e normotensos, respectivamente, eram mulheres.

Em relação a outras comorbidades ou distúrbios metabólicos $41,98 \%$ dos pacientes hipertensos tinham diabetes ( $p$-value $<0,001)$, $50,37 \%$ dislipidemias, $58,77 \%$ problemas visuais ( $p$-value $<0,001)$ e $14,57 \%$ problemas renais (Tabela 1). Dos pacientes hipertensos somente $52,8 \%$ tinham resultados de ECG e raios- $\mathrm{x}$, sendo que desses, $26,17 \%$ estavam alterados $\quad(p$-value $=0,019) . \quad$ Diferenças significativas quanto aos fatores de risco para hipertensão entre os grupos hipertenso e normotenso podem ser observadas na Tabela 1.

Em relação aos hábitos de vida, 64,52\% dos pacientes hipertensos e $100 \%$ dos normotensos informaram que fazem até três refeições ao dia ( $p$-value $=0,003)$, sendo que $40,05 \%$ dos hipertensos e $17,65 \%$ dos normotensos afirmaram que têm baixo consumo de frutas ou não as consomem. Dos hipertensos 39,55\% informaram que não seguem dieta hipossódica, mesmo sendo hipertensos, 19,75\% ingerem bebidas alcóolicas e 18,02\% são fumantes (Tabela 1).

Em relação à prática de atividade física, somente $36,54 \%$ a praticam regularmente enquanto os normotensos fazem essa atividade em $76,47 \%$ dos casos ( $p$ value $=0,001)$. 0 cálculo do índice de massa corporal dos pacientes hipertensos mostrou que $80,72 \%$ estão com sobrepeso ou obesos enquanto $52,94 \%$ dos normotensos estão classificados nessas categorias ( $p$ value $=0,013$ ). Uma medida adicional foi a circunferência abdominal, a qual foi encontrada alterada na maioria dos pacientes, tanto dos hipertensos quanto dos normotensos, correspondendo a 88,3\% e $52,94 \% \quad(p$-value $<0,001), \quad$ respectivamente (Tabela 1).

Em relação aos níveis pressóricos dos pacientes hipertensos a mediana foi de $130 \times$ $80 \mathrm{mmHg}$, dentro dos limites de normalidade. 
No entanto, os valores mínimos e máximos da PA foram de 83 e $240 \mathrm{mmHg}$ (sistólica), e de 57 e $135 \mathrm{mmHg}$ (diastólica), respectivamente. Assim, comparou-se os níveis pressóricos dos pacientes hipertensos em uso de medicação que se mantiveram acima de $140 \mathrm{mmHg} \times$ $90 \mathrm{mmHg}$ com aqueles com PA abaixo desses valores e observamos que em $61,14 \%$ $(n=247)$ dos casos a PA não estava controlada mesmo com uso da medicação. A comparação dos fatores de risco entre os pacientes hipertensos com e sem a PA controlada mostrou uma associação significativa entre circunferência abdominal alterada e hipertensos não controlados ( $p$-value $=0,002)$.

Tabela 1. Fatores de risco e hábitos de vida de pacientes hipertensos, atendidos pelo programa HIPERDIA nas Unidades Básicas de Saúde. Uberaba, agosto de 2013 a abril de 2016.

\begin{tabular}{|c|c|c|c|c|c|}
\hline \multirow[t]{2}{*}{ Fatores de risco } & \multicolumn{2}{|c|}{ Hipertensos } & \multicolumn{2}{|c|}{ Normotensos } & \multirow[t]{2}{*}{$p$-value } \\
\hline & $\mathbf{n}$ & $\%$ & $\mathbf{n}$ & $\%$ & \\
\hline Diabetes & 170 & 41,98 & 0 & 0,0 & $<0,001$ \\
\hline Problemas renais & 59 & 14,57 & 0 & 0,0 & 0,090 \\
\hline Dislipidemias & 204 & 50,37 & 6 & 35,29 & 0,223 \\
\hline Problemas visuais & 238 & 58,77 & 2 & 11,76 & $<0,001$ \\
\hline ECG/RX alterado & 56 & 26,17 & 0 & 0,0 & 0,019 \\
\hline Até três refeições ao dia & 251 & 64,52 & 16 & 100,00 & 0,003 \\
\hline Dieta sem restrição de sal & 159 & 39,55 & 6 & 35,29 & 0,725 \\
\hline $\begin{array}{l}\text { Baixo ou nenhum consumo de } \\
\text { frutas }\end{array}$ & 161 & 40,05 & 3 & 17,65 & 0,110 \\
\hline Consumo de bebida alcoólica & 80 & 19,75 & 5 & 29,41 & 0,331 \\
\hline Fumante & 73 & 18,02 & 3 & 17,65 & 0,968 \\
\hline Prática de atividade física regular & 148 & 36,54 & 13 & 76,47 & 0,001 \\
\hline Sobrepeso/Obesidade & 314 & 80,72 & 9 & 52,94 & 0,013 \\
\hline $\begin{array}{l}\text { Circunferência abdominal } \\
\text { alterada }\end{array}$ & 332 & 88,30 & 9 & 52,94 & $<0,001$ \\
\hline
\end{tabular}

Um menor percentual de indivíduos hipertensos controlados relatou fazer até três refeições ao dia ( $p$-value=0,003) e um maior percentual desse grupo seguia uma dieta sem restrição de sal ( $p$-value $=0,022)$, mostrando que outros fatores além dos hábitos alimentares devem influenciar a falta de controle da PA dos pacientes (Tabela 2). 
Tabela 2. Fatores de risco e hábitos de vida de pacientes hipertensos com pressão arterial controlada e não controlada atendidos pelo programa HIPERDIA nas Unidades Básicas de Saúde, Uberaba, agosto de 2013 a abril de 2016.

\begin{tabular}{|c|c|c|c|c|c|}
\hline \multirow[t]{2}{*}{ Fatores de Risco } & \multicolumn{2}{|c|}{$\begin{array}{l}\text { Hipertensos } \\
\text { controlados }\end{array}$} & \multicolumn{2}{|c|}{$\begin{array}{l}\text { Hipertensos } \\
\text { não } \\
\text { controlados }\end{array}$} & \multirow[t]{2}{*}{$p$-value } \\
\hline & $\mathbf{n}$ & $\%$ & $\mathbf{n}$ & $\%$ & \\
\hline Diabetes & 65 & 41,40 & 104 & 42,11 & 0,889 \\
\hline Problemas renais & 28 & 17,83 & 31 & 12,55 & 0,143 \\
\hline Dislipidemias & 84 & 53,50 & 120 & 48,58 & 0,335 \\
\hline Problemas visuais & 83 & 52,87 & 154 & 62,35 & 0,059 \\
\hline ECG/RX alterado & 23 & 30,26 & 33 & 24,09 & 0,327 \\
\hline Até três refeições ao dia & 82 & 55,41 & 169 & 70,42 & 0,003 \\
\hline Dieta sem restrição de sal & 72 & 46,75 & 87 & 35,22 & 0,022 \\
\hline $\begin{array}{l}\text { Baixo ou nenhum consumo de } \\
\text { frutas }\end{array}$ & 69 & 44,81 & 91 & 36,84 & 0,139 \\
\hline Consumo de bebida alcoólica & 27 & 17,20 & 52 & 21,05 & 0,341 \\
\hline Fumante & 31 & 19,75 & 42 & 17,00 & 0,486 \\
\hline Prática de atividade física regular & 58 & 36,94 & 89 & 36,03 & 0,853 \\
\hline Sobrepeso/Obesidade & 117 & 78,00 & 197 & 82,43 & 0,344 \\
\hline $\begin{array}{l}\text { Circunferência } \\
\text { alterada }\end{array}$ & 116 & 81,69 & 215 & 92,27 & 0,002 \\
\hline
\end{tabular}

\section{DISCUSSÃO}

0 trabalho realizado nas UBS foi bem aceito pelas Equipes de Saúde da Família (ESF), que incluíram o tema da pesquisa na atividade didática do HIPERDIA, mostrando que programas de capacitação, como este, são importantes para incentivar os profissionais de saúde e promover um atendimento de melhor qualidade.

No entanto, não houve grande adesão por parte dos pacientes $(18,4 \%)$, sendo vários os motivos elencados por eles, como: medo de coletar sangue ou descobrir alguma doença, ter que voltar rápido para casa para cuidar do companheiro doente ou dos netos, trabalho, entre outros. Os locais de maior adesão foram aqueles que tiveram apoio e incentivo por parte da equipe de enfermagem, na qual os pacientes têm confiança.

A maioria das pessoas hipertensas tinha idade superior a 51 anos, como esperado. No entanto, pacientes de 18-50 anos também participaram do estudo e representaram um número expressivo, $23,7 \% \quad(n=96)$, confirmando que a HAS é um grave problema de saúde pública, uma vez que atinge pessoas em fase produtiva da vida. A heterogeneidade do grupo hipertenso, em relação à idade, pode ser explicada parcialmente pelo fato das ESF realizarem busca ativa dos pacientes, encaminhando os mesmos para atendimento nas unidades de saúde conforme cada caso ${ }^{8}$.

A maior participação do gênero feminino corrobora outros estudos ${ }^{9-11}$. Numa pesquisa com o HIPERDIA, houve participação de $69,4 \%$ de mulheres ${ }^{9}$. Em outro estudo para avaliar a relação da atividade física e índices antropométricos de hipertensos e diabéticos, $66,25 \%$ foi do sexo feminino ${ }^{10}$. Ainda, em outra pesquisa para associar fatores de risco e complicações em hipertensos e diabéticos, a participação de mulheres foi de 73,6\% ${ }^{11}$. Estes dados indicam que a mulher procura mais pelas unidades de saúde, inclusive para acompanhamento do tratamento, mostrando maior preocupação com sua saúde.

0 diabetes foi uma comorbidade frequente e significativa nos pacientes hipertensos, com índice um pouco maior $(41,97 \%)$ do que encontrado em trabalhos similares $31 \% 9$ e $33,5 \%{ }^{12}$ e mais próximos dos índices das 7aㅡ Diretriz Brasileira de Hipertensão Arterial $^{4}$, que colocam a 
possibilidade de associação do diabetes e da hipertensão em torno de 50\%.

Tal associação é preocupante, uma vez que as duas doenças têm mecanismos de lesão micro e macrovascular que podem levar a acidentes cardiocerebrovasculares importantes 4 . Os hipertensos não controlados tinham diabetes na mesma proporção que os hipertensos controlados (42,11\% e 41,40\%, respectivamente), dado que contrasta com outro estudo que mostrou uma associação direta entre hipertensos não controlados e diabetes ${ }^{9}$.

Outra associação significativa que foi observada neste estudo foi em relação aos problemas visuais, mais observados em pacientes hipertensos do que nos normotensos. Além disso, também foi maior em pacientes hipertensos não controlados, mesmo com uso de medicação, do que naqueles controlados, apesar de não haver significância estatística.

Problemas renais foram observados em $14,57 \%$ dos pacientes hipertensos, mas sem significância estatística. Talvez o baixo índice de complicações renais seja em função do acompanhamento dos pacientes pelo programa HIPERDIA, uma vez que a hipertensão é reconhecidamente o fator de risco mais importante para a progressão da lesão renal em população diabética ou não ${ }^{13}$.

Em relação aos hábitos de vida houve associação significativa do sobrepeso e obesidade, medidas de circunferência abdominal, sedentarismo e o consumo de alimentos até três vezes por dia com pacientes hipertensos.

Nessa população o consumo de bebidas alcóolicas e de tabaco foi de 19,75\% e 18,02\%, mas sem associação significativa com a hipertensão. Estes dados estão de acordo com outros estudos que mostram que a obesidade, o sedentarismo e o aumento da circunferência abdominal são grandes fatores de risco, não só para a HAS, mas também para acidentes vasculares cerebrais isquêmicos e outros ${ }^{10-}$ 12,14 .

Por outro lado, já é amplamente difundido que a prática de atividade física auxilia, não só na prevenção da HAS, como no tratamento não-medicamentoso e no controle dos níveis pressóricos ${ }^{15,16}$.

$\mathrm{Na}$ análise dos fatores de risco entre os hipertensos com a PA controlada e os não controlados foi observado que estes últimos tinham maiores medidas de circunferência abdominal ( $p$-value $=0,002) . \quad$ No entanto, nenhuma associação foi encontrada em relação ao sedentarismo e ao sobrepeso e a obesidade, nem quanto ao controle inadequado da PA.

A maior proporção dos hipertensos não controlados fazia até três refeições por dia e não se preocupava em manter uma dieta hipossódica, o que demonstrou que, apesar da dieta hipossódica ser fundamental para auxiliar no controle da PA, existem outros fatores, como os genéticos e a baixa adesão ao tratamento, que são responsáveis por manter a PA alterada.

$\mathrm{O}$ controle inadequado da PA tem sido observado $^{9}$, o que sugere que o programa HIPERDIA, apesar de contribuir com a redução dos acidentes vasculares cerebrais no Brasil6, precisa melhorar a estratégia de sensibilização da população para o cuidado com sua saúde e estimular e capacitar melhor os agentes de saúde para uma ação adequada junto à população.

\section{CONCLUSÃO}

Este estudo permitiu a identificação de fatores de risco associados aos usuários do HIPERDIA, como diabetes, problemas visuais e cardíacos (ECG e raios-x alterados), difíceis de serem modificados, e outros fatores, tais como o sedentarismo, o sobrepeso/obesidade, o aumento da circunferência abdominal e o controle da pressão arterial, que poderiam ser reduzidos com uma boa adesão dos usuários às práticas incentivadas pelo Programa.

Isso mostra que, apesar das ações do HIPERDIA serem importantes, elas não têm conseguido conscientizar uma parte dos usuários a tomarem medidas que possam reduzir as complicações da HAS, associadas ou não ao diabetes. 


\section{REFERÊNCIAS}

1. Ministério da Saúde (Br). Secretaria de Atenção à Saúde, Departamento de Atenção Básica. Vigilância em saúde: zoonoses. Brasília: Ministério da Saúde; 2009.

2. Nogueira D, Faerstein E, Coeli CM, Chor D, Lopes CDS, Werneck GL. Reconhecimento, tratamento e controle da hipertensão arterial: estudo pró-saúde, Brasil. Rev Panam Salud Publica. 2010; 27(2):103-9.

3. Daniel ACQG, Veiga EV. Fatores que interferem na adesão terapêutica medicamentosa em hipertensos. Einstein. 2013; 11(3):331-7.

4. Malachias MVB, Souza WKSB, Plavnik FL, Rodrigues CIS, Brandão AA, Neves MFT, et al. 7aㅡ Diretriz Brasileira de Hipertensão Arterial. Arq Bras Cardiol. 2016; 107(3 Supl 3):1-83.

5. Passos VMDA, Assis TD, Barreto SM. Hipertensão arterial no Brasil: estimativa de prevalência a partir de estudos de base populacional. Epidemiol Serv Saúde. 2006; 15(1):35-45.

6. Lopes JM, Sanchis GJB, Medeiros JLAD, Dantas FG. Hospitalização por acidente vascular encefálico isquêmico no Brasil: estudo ecológico sobre possível impacto do HIPERDIA. Rev Bras Epidemiol. 2016; 19(1):122-34.

7. Prefeitura Municipal (Uberaba, MG), Secretaria de Saúde. Hiperdia Uberaba/MG [Internet]. 2017 [citado em: 3 maio 2017]. Disponível em: http: uberaba.mg.gov.br/portal

8. Kebian LVA, Acioli S. A visita domiciliar de enfermeiros e agentes comunitários de saúde da Estratégia Saúde da Família. Rev Eletrônica Enferm. [Internet]. 2014 [citado em: 3 maio 2017]; 16(1):161-9. Disponível em: https://www.fen.ufg.br/revista/v16/n1/pdf /v16n1a19.pdf DOI: http://dx.doi.org/10.5216/ree.v16i1.20260 9. Souza CS, Stein AT, Bastos GAN, Pellanda LC. Controle da pressão arterial em hipertensos do Programa HIPERDIA: estudo de base territorial. Arq Bras Cardiol. 2014; 102(6):571-8.

10. Cunha RM, Souza CODS, Silva JFD, Silva MAD. Nível de atividade física e índices antropométricos de hipertensos e/ou diabéticos de uma cidade do Brasil. Rev Salud Publica. 2012:429-37.

11. Santos JC, Moreira TMM. Fatores de risco e complicações em hipertensos/diabéticos de uma regional sanitária do nordeste brasileiro. Rev Esc Enferm USP. 2012; 46(5):1125-32.

12. Cabral NAL, Ribeiro VS, França AKTC, Salgado JVL, Santos AM, Salgado Filho N, et al. Cintura hipertrigliceridêmica e risco cardiometabólico em mulheres hipertensas. Rev Assoc Med Bras. 2012; 58(5):568-73.

13. Moreira HG, Sette JBC, Keiralla LCB, Alves SG, Pimenta E, Sousa $M$, et al. Diabetes mellitus, hipertensão arterial e doença renal crônica: estratégias terapêuticas e suas limitações. Rev Bras Hipertens. 2008; 15(2):111-6.

14. Montenegro-Neto AN, Silva-Simões MO, Medeiros ACD, Portela AS, Queiroz MSR, Cunha-Montenegro $\mathrm{R}$, et al. The correlation between anthopometric measurements and biochemical cardiovascular risk markers in the hypertensive elderly. Rev Salud Publica. 2011; 13(3):421-32.

15. Fisher MM. The effect of resistance exercise on recovery blood pressure in normotensive and borderline hypertensive women. J Strength Cond Res. 2001; 15(2):2106.

16. Vicent KR, Braith RW. Resistance and bone turnover in elderly men and women. Med Sci Sports Exerc. 2002; 34(1):17-23.

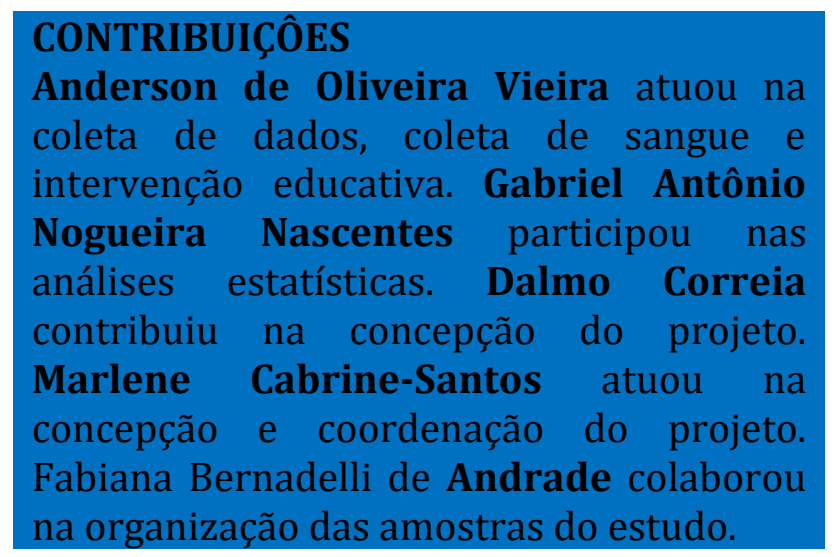




AGRADECIMENTOS
Aos servidores da Universidade Federal do
Triângulo Mineiro: Oberdan Ricardo
Ribeiro, Ana Carolina de Oliveira Moraes e
Sílvia Baldan Costa pelo auxílio nas coletas
de sangue e preenchimento dos
questionários.
Aos órgãos de fomento, FAPEMIG (APQ-
$00155 / 14)$ e CNPq (Processo
$448633 / 2014-0)$ pelo apoio financeiro.

\footnotetext{
Como citar este artigo (Vancouver)

Vieira AO, Nascentes GAN, Correia D, Santos MC. Avaliação das ações do programa Hiperdia para pacientes hipertensos. REFACS [Internet]. 2018 [citado em: inserir dia, mês e ano de acesso]; 6(3): 445-452. Disponível em: inserir link de acesso. DOI: inserir link do DOI.

\section{Como citar este artigo (ABNT)}

VIEIRA, A. O. et al. Avaliação das ações do programa Hiperdia para pacientes hipertensos. REFACS, Uberaba, MG, v. 6, n. 3, p. 445-452, 2018. Disponível em: <inserir link de acesso>. Acesso em: inserir dia, mês e ano de acesso. DOI: inserir link do DOI.

Como citar este artigo (APA)

Vieira, A. O., Nascentes, G. A. N., Correia, D. \& Santos, M. C. (2018). Avaliação das ações do programa Hiperdia para pacientes hipertensos. REFACS, 6(3), 445-452. Recuperado em: inserir dia, mês e ano de acesso de inserir link de acesso. DOI: inserir link do DOI.
} 